\title{
Delayed Cord Clamping in Infants of Diabetic Mothers: Laboratory and Clinical Outcomes
}

\author{
Shaimaa Reda Abdelmaksoud ${ }^{10}$ Heba Elsayed Abdelraziq ${ }^{2}$ Rana Atef khashaba ${ }^{3(0)}$ \\ Aliaa Mohamed Diab ${ }^{1}$ \\ ${ }^{1}$ Department of Pediatrics, Benha Faculty of Medicine, Benha \\ University, Benha, Egypt \\ ${ }^{2}$ Department of Obstetrics and Gynecology Benha Faculty of \\ Medicine, Benha University, Benha, Egypt \\ ${ }^{3}$ Department of Clinical Pathology and Chemistry, Benha Faculty of \\ Medicine, Benha University, Benha, Egypt \\ Address for correspondence Shaimaa Reda Abdelmaksoud, MD, \\ Department of Pediatrics, Benha Faculty of Medicine, Benha \\ University, Benha, Egypt (e-mail: shaimaareda82@gmail.com). \\ J Child Sci 2021;11:e74-e79.
}

\begin{abstract}
Keywords

- delayed cord clamping

- infants of diabetic mothers

- polycythemia

The aim of this study was to compare the effects of delayed cord clamping (DCC) and early cord clamping (ECC) on venous hematocrit (htc) and clinical outcomes in infants of diabetic mothers (IDMs). This prospective randomized study included 157 term IDMs. The umbilical cords of these infants were clamped at least 60 seconds in group I (DCC group, $n=79$ ) and as soon as possible after birth in group II (ECC group, $n=78$ ). The two groups were compared regarding neonatal venous htc levels, hypoglycemia rates, jaundice requiring phototherapy, respiratory distress, and admission to the neonatal intensive care unit (NICU). Hematocrit levels were significantly higher in the DCC group, both at 6 and 24 hours postnatally $(p=0.039$ and 0.01$)$, respectively. Polycythemia frequency was higher in DCC than the ECC group, but no patient in either group needed partial exchange transfusion (PET). Rates of jaundice were significantly higher in the DCC group ( $p=0.028)$, but there was no significant difference between the two groups regarding jaundice requiring phototherapy $(p=0.681)$. There were no differences between the groups regarding hypoglycemia rates, need for glucose infusion, or respiratory distress. The incidence of admission to NICU was lower in the DCC group $(p=0.005)$. Early clamping was a significant predictor for increased risk of NICU admission. DCC increased polycythemia and jaundice rates but did not increase the need for PET or phototherapy. Also, DCC reduced the severity of respiratory distress and the subsequent need for NICU admission.
\end{abstract}

\section{Introduction}

The optimal timing of umbilical cord clamping remains controversial. ${ }^{1}$ Umbilical cord clamping can be done immediately after birth or be delayed. Delayed cord clamping (DCC) can be performed by clamping the cord from 30 seconds to 3 minutes after birth when cord pulsation has ceased, and the infant has started crying. ${ }^{2-4}$

received

December 8, 2020

accepted after revision

February 18, 2021
DOI https://doi.org/

10.1055/s-0041-1726460.

ISSN 2474-5871.
It has been shown that DCC can increase placental transfusion, leading to an increase in neonatal blood volume at birth of approximately $30 \%$. In a term infant, this may result in an increase in iron stores, thereby decreasing the risk of anemia. ${ }^{5}$ DCC, however, might increase the risk of polycythemia, jaundice, the need for phototherapy, and respiratory distress, especially in certain risk groups of infants. ${ }^{6}$ DCC is also associated with

(C) 2021. The Author(s).

This is an open access article published by Thieme under the terms of the Creative Commons Attribution License, permitting unrestricted use, distribution, and reproduction so long as the original work is properly cited. (https://creativecommons.org/licenses/by/4.0/) Georg Thieme Verlag KG, Rüdigerstraße 14, 70469 Stuttgart, Germany 
improved developmental milestones at infancy until 4 years of age. ${ }^{7}$

It has been shown that about 6 to $9 \%$ of pregnancies are complicated by diabetes and approximately $90 \%$ of these cases represent women with gestational diabetes mellitus (GDM). GDM is a condition in which carbohydrate intolerance occurs during pregnancy. 8,9

Infants born to mothers with diabetes are at a greater risk of polycythemia, which is associated with hyperviscosity and hyperbilirubinemia. ${ }^{10}$

IDMs are at significantly greater risk of developing DCC complications, such as polycythemia, respiratory distress, and hyperbilirubinemia. ${ }^{11}$ Although many studies have shown that DCC did not increase the risk of polycythemia in term infants, the significantly increased rate of polycythemia in IDMs raises concerns regarding this group's intervention. ${ }^{12}$ For these reasons, IDMs have been excluded from the previously conducted DCC studies, and there is a lack of data about the laboratory and clinical outcomes related to the effects of DCC in the group of IDMs. Therefore, the safety and effects of DCC in IDMs are unknown.

This study's primary outcome was to analyze the effects of DCC on the incidence of polycythemia and hematocrit (htc) levels in IDMs. Secondary outcomes were to reveal its effects on rates of hypoglycemia, hypocalcemia, respiratory distress, the NICU admission, and jaundice requiring phototherapy.

\section{Patients and Methods}

\section{Participants}

This prospective randomized clinical trial was designed to evaluate DCC's effects early in the postnatal period in IDM. The trial was conducted in accordance with ethical principles that had their origin in the Declaration of Helsinki. This clinical trial was reviewed and approved by the Institutional Ethical Committee (approval number: RC10-9-2019). Written informed consent was obtained from the parents of the neonate who were included in the study.

Pregnant women were eligible for this study, along with their babies, if they had diabetes (type 1, type 2, or GDM) and gave birth at or later than 37 weeks of gestation. Infants with a major congenital malformation, fetal hydrops, congenital infection, and multiple births were excluded from the study. We also excluded neonates without informed parental consent. In addition, neonates who required resuscitation after birth were also excluded.

\section{Sample Size Estimation}

The sample size was calculated by using power and sample size software version 3.0. Based on a previous study done by Korkut et al, ${ }^{13}$ the percentage of neonates who needed NICU admission was $27.5 \%$ in the early clamping group compared with $10.0 \%$ in the delayed clamping group. The total sample size of 154 ( 77 per group) will be sufficient to detect power of $80 \%$ and a significance level of $5 \%$. Considering possible dropouts during the study, a decision was made to randomize 85 subjects to each group.

\section{Randomization and Blinding}

Infants enrolled in this study were randomly assigned to two interventional groups: group I included infants for DCC, and group II included infants for ECC (simple 1:1 randomization). The randomization was done 10 minutes before delivery by using the sealed, numbered envelopes containing the treatment allocations. Blinding was not feasible for this study because of the nature of the procedure.

\section{Intervention}

DCC was performed by clamping the cord at least 60 seconds after the infant was born. On the other hand, in the ECC group, clamping of the cord was done as soon as possible after birth. Infants who underwent DCC were held during the waiting period at the level of mothers' abdomens. ${ }^{14}$ The cord blood samples were collected from the umbilical vein after cord clamping from the baby's side. No additional interventions were performed.

\section{Management of Infant of Diabetic Mothers}

All healthy newborns were breastfed within 1 hour of birth and then the initial blood glucose levels were recorded 30 minutes after feeding. They fed every 2 to 3 hours, and their prefeed blood glucose levels were measured for 12 hours postnatal. The infants were exclusively breastfed whenever possible. Hypoglycemia was defined as blood glucose levels of $<40 \mathrm{mg} / \mathrm{dL}$ in first 4 hours postnatal and $<45 \mathrm{mg} / \mathrm{dL}$ from 4 to 24 hours after birth. The blood glucose levels of hypoglycemic infants were checked 1-hour postfeed. In cases of recurrent hypoglycemia, the baby is fed again or administrated IV glucose. In severe hypoglycemia, defined as decreased blood glucose level less than $25 \mathrm{mg} / \mathrm{dL}$ in first 4 hours postnatal or less than $30 \mathrm{mg} / \mathrm{dL}$ in 4 to 24 hours, they were immediately administered IV glucose. ${ }^{15}$ The infants administrated IV glucose were admitted to NICU.

The cord, postnatal 6 hours, and postnatal 24 hours htc levels were measured for all enrolled infants. Polycythemia was defined as venous htc $>65 \% .^{16}$ PET was indicated for symptomatic polycythemic infants and asymptomatic polycythemic infants with htc $>75 \%$. Infants and the need for PET were considered an indication for NICU admission.

\section{Blood Tests}

Htc levels were measured by collecting samples of venous blood in heparinized capillary tubes and then centrifugation of these tubes was done for 5 minutes before htc levels measurement. Monitoring of capillary blood glucose levels was done by using quick strips of the Roche Accu-check Inform II glucometer device. Confirmation of hypoglycemia was done by the measurement of plasma glucose concentration. Plasma glucose levels were determined by using the Roche Hitachi Cobas 6000 auto-analyzer. Its measuring system is based on electrochemiluminescence (ECL) principles.

\section{Maternal and Neonatal Characteristics}

The data on the gender, gestational age, birth weight, length, head circumference if the infant was large for gestational age 
(LGA; defined as birth weight above the 90th percentile for their gestational age and gender), ${ }^{17} 5$-minute Apgar's scores, htc levels at 6 and 24 hours postanal, plasma glucose levels, presence of jaundice requiring intervention, levels of serum calcium at 24 hours after birth, the presence of respiratory distress ( $R R>60$ breath per minute, nasal flaring, intercostal and/or suprasternal retractions, or need oxygen therapy), and NICU admission was recorded. The enrolled infants were followed either with their mothers or in NICU according to their medical conditions. The infants who developed postnatal problems such as feeding problems or respiratory distress underwent close monitoring for a maximum 6 hours, either returned to their mother or admitted to NICU depending on their clinical condition. Infants were followed up for jaundice and the need for phototherapy postnatally for 10 days. ${ }^{18}$ Maternal data such as maternal age, maternal weight, type of diabetes, diseases, medication, and mode of delivery were obtained and recorded.

\section{Results}

Recruitment was conducted from September 2019 to August 2020. As shown in -Fig. 1, 170 IDM eligible for this study were enrolled. After exclusion and the follow-up period, we finally analyzed 79 neonates in the DCC group and 78 neonates in ECC group.

The mothers and infants in both groups were similar with respect to demographic and clinical characteristics - Table 1 .

The cord 6-hour postnatal and 24-hour postnatal htc values were compared in -Table $\mathbf{2}$, and there was no significant difference between cord htc values in both groups. Neonatal htc at 6 hours of age in the DCC group was significantly higher than in the ECC group $(p=0.039)$. At 24 hours, postnatal htc value in the DCC group was decreased but still significantly higher than those of the ECC group $(p=0.01)$.

Laboratory and clinical features of both cohorts are presented in - Table 3. The incidence of polycythemia in the DCC group was $41.8 \%$ at 6 hours and $29.1 \%$ at 24 hours, while in the ECC group, it was $14.1 \%$ at 6 hours and $5.1 \%$ at 24 hours. Rates of polycythemia were significantly higher in the DCC group than those of the ECC group, both at 6 and 24 hours. postnatally, $(p<0.001)$. Despite that, none of the infants in either group underwent partial exchange transfusion.

There were no significant differences between two groups regarding hypoglycemia, the need for glucose infusion, respiratory distress, and hypocalcemia. After birth, the mean calcium level at 24 hours was found to be $8.7 \pm 0.7 \mathrm{mg} / \mathrm{dL}$ in

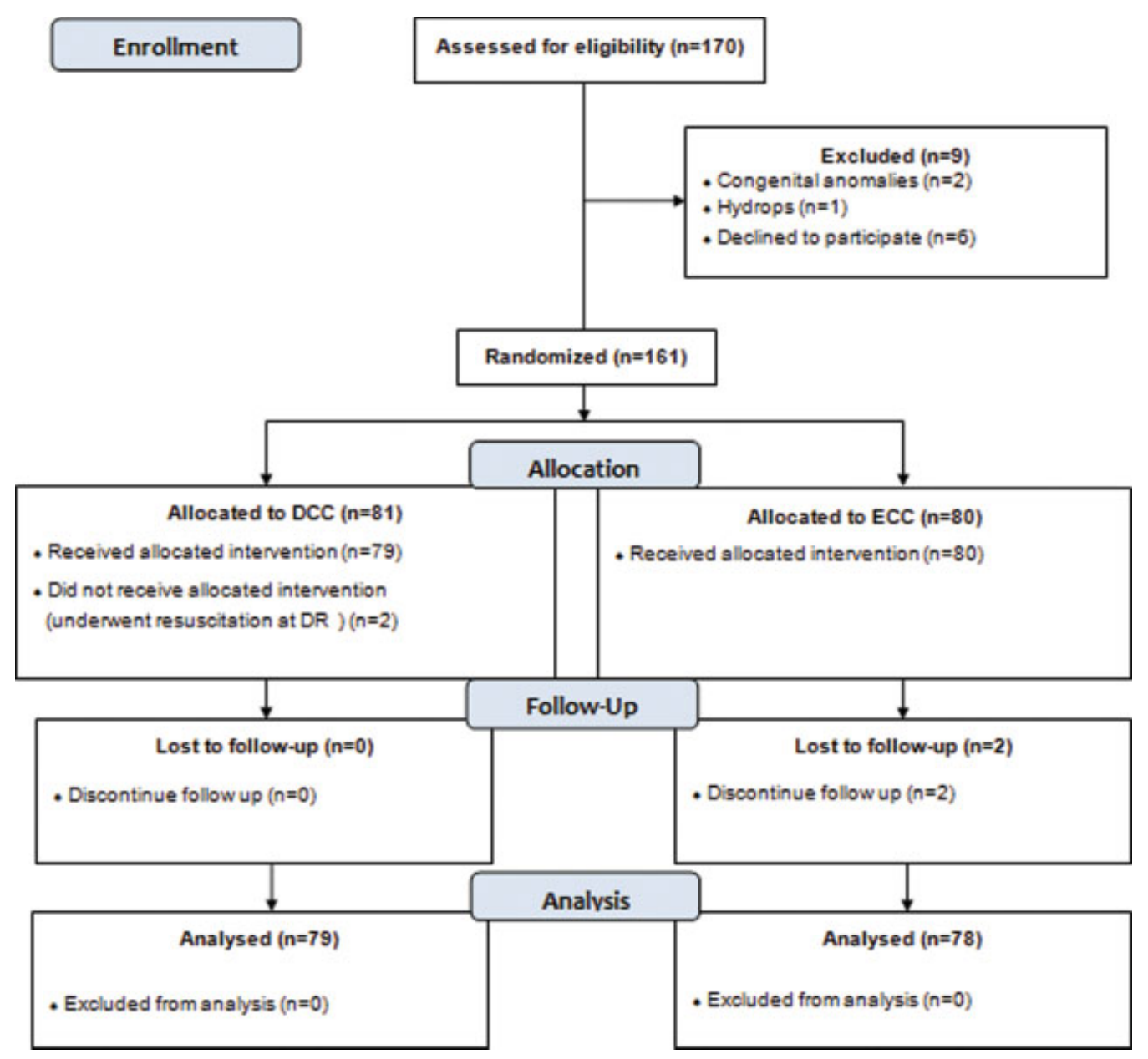

Fig. 1 CONSORT flow diagram of study participants. 
Table 1 Maternal and infantile baseline characteristics

\begin{tabular}{|c|c|c|c|c|}
\hline & & $\begin{array}{l}\text { Delayed clamping } \\
(n=79)\end{array}$ & $\begin{array}{l}\text { Early clamping } \\
(n=78)\end{array}$ & $p$-Value \\
\hline Maternal age (y) & Mean \pm SD & $28.7 \pm 5.5$ & $28.4 \pm 5.1$ & 0.759 \\
\hline Maternal weight $(\mathrm{kg})$ & Mean \pm SD & $81.5 \pm 7.9$ & $81.7 \pm 8.2$ & 0.91 \\
\hline \multirow[t]{3}{*}{ Type of diabetes, $n$ (\%) } & Gestational & $71(89.9)$ & $71(91.0)$ & \multirow[t]{3}{*}{0.839} \\
\hline & Type I & $2(2.5)$ & $3(3.8)$ & \\
\hline & Type II & $6(7.6)$ & $4(5.1)$ & \\
\hline Cesarean section, $n(\%)$ & Yes & $55(69.6)$ & $46(59.0)$ & 0.164 \\
\hline \multirow[t]{2}{*}{ Gender, $n(\%)$} & Males & $37(46.8)$ & $39(50.0)$ & \multirow[t]{2}{*}{0.692} \\
\hline & Females & $42(53.2)$ & $39(50.0)$ & \\
\hline Birth weight (g) & Mean \pm SD & $3,738.5 \pm 270.2$ & $3,728.5 \pm 271.5$ & 0.817 \\
\hline Apgar's score & Median (range) & $8(7-10)$ & $8(7-10)$ & 0.487 \\
\hline Length $(\mathrm{cm})$ & Mean \pm SD & $48.6 \pm 1$ & $48.6 \pm 1.1$ & 0.631 \\
\hline Gestational age (wk) & Mean \pm SD & $38.1 \pm 0.7$ & $38.1 \pm 0.7$ & 0.855 \\
\hline LGA, $n(\%)$ & Yes & $21(26.6)$ & $20(25.6)$ & 0.893 \\
\hline Head circumference $(\mathrm{cm})$ & Mean \pm SD & $35.2 \pm 1.7$ & $35.2 \pm 1.7$ & 0.993 \\
\hline
\end{tabular}

Abbreviations: LGA, Large for gestational; SD, standard deviation.

Note: Independent $t$-test was used for numerical data. Mann-Whitney U test was used for Apgar's score. Chi-square test was used for categorical data.

Table 2 Hematocrit values of infants

\begin{tabular}{|l|l|l|l|l|}
\hline & \multicolumn{2}{|l|}{$\begin{array}{l}\text { Delayed clamping } \\
(\boldsymbol{n = 7 9 )}\end{array}$} & $\begin{array}{l}\text { Early clamping } \\
(\boldsymbol{n}=78)\end{array}$ \\
\hline Hematocrit (\%) & Mean \pm SD & $55.5 \pm 7.4$ & $53.7 \pm 9.7$ & 0.207 \\
\hline Cord & Mean \pm SD & $59.7 \pm 8.2$ & $57 \pm 7.6$ & 0.039 \\
\hline 6-h postnatal & Mean \pm SD & $57.2 \pm 8.4$ & $53.9 \pm 7.3$ & 0.01 \\
\hline 24-h postnatal &
\end{tabular}

Abbreviation: SD, standard deviation.

Note: Independent $t$-test was used.

Table 3 Clinical and laboratory features of infants

\begin{tabular}{|c|c|c|c|}
\hline & $\begin{array}{l}\text { Delayed clamping } \\
(n=79)\end{array}$ & $\begin{array}{l}\text { Early clamping } \\
(n=78)\end{array}$ & $p$-Value \\
\hline \multicolumn{4}{|l|}{ Polycythemia, $n$ (\%) } \\
\hline 6-h postnatal & $33(41.8)$ & $11(14.1)$ & $<0.001$ \\
\hline 24-h postnatal & $23(29.1)$ & $4(5.1)$ & $<0.001$ \\
\hline Hypoglycemia, n (\%) & $12(15.2)$ & $17(21.8)$ & 0.286 \\
\hline Need glucose infusion, $n(\%)$ & $1(1.3)$ & $2(2.6)$ & 0.62 \\
\hline Respiratory distress, $n(\%)$ & $26(32.9)$ & $23(29.5)$ & 0.643 \\
\hline Need NICU, $n(\%)$ & $9(11.4)$ & $23(29.5)$ & 0.005 \\
\hline Jaundice, $n(\%)$ & $25(31.6)$ & $13(16.7)$ & 0.028 \\
\hline Need phototherapy, $n(\%)$ & $4(5.1)$ & $2(2.6)$ & 0.681 \\
\hline Hypocalcemia, $n$ (\%) & $9(11.4)$ & $11(14.1)$ & 0.611 \\
\hline
\end{tabular}

Abbreviation: NICU, neonatal intensive care unit.

Note: Chi-square or Fisher's exact test was used for categorical data. 
the DCC group and $8.6 \pm 0.7 \mathrm{mg} / \mathrm{dL}$ in the ECC group. There was no statistically significant difference between the groups in terms of calcium levels $(p=0.471)$.

The incidence of NICU admission in the ECC group was significantly higher than DCC group, as 23 out of 78 patients (29.5\%) in the ECC group need NICU admission compared with only 9 out 79 patients (11.4\%) in the DCC group who need NICU admission ( $p=0.005)$.

Eight of the nine patients in the DCC group who were admitted to NICU were diagnosed with respiratory distress, and one patient was monitored for hypoglycemia. Four of them required phototherapy during admission. In the ECC group, 21 infants were monitored in NICU for respiratory distress, and two infants for hypoglycemia. Among these 23 patients, only two infants needed phototherapy.

Although the patients who developed respiratory distress (26 infants) in the DCC group were more than those in the ECC group (23 infants), the rate of NICU admission due to respiratory distress was $8 / 26(30.8 \%)$ in the DCC group and $21 / 23(91.3 \%)$ in the ECC group $(p<0.001)$.

The incidence of jaundice was $31.6 \%$ in the DCC group and $16.7 \%$ in the ECC group. Jaundice rates were significantly higher in the DCC group than in the ECC group $(p=0.028)$. Despite the high incidence rate of jaundice in the DCC group, only 4/79 patients in the DCC group need phototherapy than 2/78 patients in ECC. The difference, however, was not found to be statistically significant.

On logistic regression analysis, after adjustment for maternal age, maternal weight, type of delivery, gender, gestational age, and birth weight, early clamping was a significant predictor for increased risk of NICU admission three times more than delayed clamping (odd ratio $=3.242 ; 95 \%$ confidence interval $=1.364-7.705 ; p=0.008)$.

\section{Discussion}

To our knowledge, there is a lack of studies that discuss the effect of DCC in IDMs. This group has been excluded from DCC studies due to the risk of complications.

Our results showed that DCC in IDMs increased the incidence of polycythemia but without the need for PET. Rates of NICU admission were higher in the ECC than DCC group. A high proportion of infants with respiratory distress in the ECC group needed admission to NICU. DCC performance did not affect rates of hypoglycemia or jaundice requiring phototherapy.

In 2019, Korkut et $\mathrm{al}^{13}$ studied the effects of DCC on 40 IDMs. They calculated the sample size depending on rates of polycythemia as a primary outcome, but in our study, we calculated sample size based on the indication for NICU admission. We took into account secondary effects such as hypoglycemia, jaundice requiring phototherapy, and hypocalcemia.

The newborn htc peaks in the second hour of age, gradually decrease, and generally stabilizes within 12 to 24 hours. The initial increase is associated with the transudation of fluid out of the intravascular space. ${ }^{19}$ Our results are consistent with this physiological state in the newborn. The htc levels were higher at 6 hours postnatally in both groups and declined at 24 hours postnatally.

In our study, htc levels were significantly higher in infants allocated to DCC at 6 and 24 hours postnatally. This is in agreement with the study by Hutton and Hassan, ${ }^{1}$ who included in their study term infants who did not have any additional risk for complications. In contrast to the results of a previous study conducted to detect the effects of DCC on LGA, which reported that there was no statistically significant difference between the DCC and ECC groups in terms of htc levels measured at the second hour of age. ${ }^{6}$

The incidence of polycythemia has been demonstrated to be between 10 and $40 \%$ in IDM. ${ }^{20}$ A previous study evaluating DCC in term newborns indicated that DCC increases htc levels in term infants but does not change polycythemia incidence. ${ }^{2}$ Rincón et al reported that DCC increases rates of polycythemia without increasing rates of PET. ${ }^{21}$ Similarly, in our study, DCC significantly increased polycythemia incidence at postnatal 6 and 24 hours compared with the ECC group. However, none of the babies required PET for polycythemia.

In our study, although the rates of clinical jaundice were significantly higher in DCC than in the ECC group, 31.6 and $16.7 \%$, respectively, the need for phototherapy rates were approximately similar in both groups. A previous study showed that DCC does not influence the rates of clinical jaundice. It was 17 and 14\% in DCC and ECC groups, respectively. ${ }^{22}$ This difference can be explained by the fact that our study was conducted on IDMs only. Our results are in agreement with two previous studies that showed that DCC does not increase the need for phototherapy. ${ }^{6,13}$ However, Chaparro et al demonstrated that the need for phototherapy was higher in infants with DCC than in infants with ECC. ${ }^{22}$

In our study, rates of hypoglycemia, hypoglycemia requiring glucose infusion, and hypocalcemia were all higher in the ECC group than in the DCC group, but there was also no statistically significant difference. Our results are approximately similar to a previous study, ${ }^{13}$, but they did not discuss hypocalcemia in their results.

Respiratory distress and subsequent NICU admission are encountered more frequently in LGA infants and IDMs than in term average for gestational age newborns. ${ }^{10}$ Our study revealed that respiratory distress incidence was higher in DCC than ECC group, but without significant difference. Despite this, respiratory distress was relatively more severe in the ECC group, and most of the infants who developed respiratory distress in the ECC group needed NICU admission. The relatively mild respiratory distress in the DCC group may be due to DCC's beneficial effect on postnatal cardiovascular adaptation. As a facilitator of adaptation, DCC decreased the rates of NICU admission by $18.1 \%$ in IDMs. In contrast to our results, a previous study reported that respiratory distress was not observed in the ECC group; however, three infants in the DCC group were admitted to the NICU due to transient tachypnea, but this difference was not significant, ${ }^{6}$ the relatively smaller sample included in their study may explain this discrepancy.

Notably, in our study, the incidence of NICU admission was significantly higher in the ECC group than in the DCC group 
$(p=0.005)$. A meta-analysis by Hutton and Hassan ${ }^{1}$ demonstrated that DCC did not increase the possibility of NICU admission in term infants who were not at risk. Notably, these studies did not include this high-risk group (IDMs). A previous study evaluated the potential placental transfusion effects on the newborn at three different cord-clamping time intervals, reported that delaying cord clamping 1 minute in term infants increases NICU admission ratios (5.5 vs. $4.3 \%$ ). In contrast, a delay of 3 minutes results in a further increase ( $8.7 \mathrm{vs.} 4.3 \%){ }^{23}$ Although there was a difference in the three groups' results, it did not rise to be statistically significant.

Regarding the 5 minutes of Apgar's score, previous studies could not determine any significant difference between the DCC and ECC groups. ${ }^{2,24}$ Similarly, we did not demonstrate any statistically significant difference between the groups in terms of the Apgar's scores, and all the infants had Apgar's scores $\geq 7$.

We have documented that ECC was an independent predictor of NICU admission in infants born to diabetic mothers on multivariate logistic regression analysis. But further studies with a larger sample size are needed to evaluate such findings.

\section{Conclusion}

DCC for 60 seconds in IDMs increased the incidence of polycythemia but did not increase the need for PET. DCC increased rates of jaundice but did not result in a greater need for phototherapy. The severity of respiratory distress and the subsequent need for NICU admission is significantly reduced by DCC. Although ECC was found to be an independent predictor of NICU admission in IDMs, studies with adequate sample size are needed to conclusively demonstrate the relation between ECC and rates of NICU admission in this cohort.

\section{Funding \\ None.}

\section{Conflict of Interest}

None declared.

\section{References}

1 Hutton EK, Hassan ES. Late vs early clamping of the umbilical cord in full-term neonates: systematic review and meta-analysis of controlled trials. JAMA 2007;297(11):1241-1252

2 McDonald SJ, Middleton P, Dowswell T, Morris PS. Effect of timing of umbilical cord clamping of term infants on maternal and neonatal outcomes. Cochrane Database Syst Rev 2013;2013 (07):CD004074

3 Roehr CC, Hansmann G, Hoehn T, Bührer C. The 2010 Guidelines on Neonatal Resuscitation (AHA, ERC, ILCOR): similarities and differences-what progress has been made since 2005? Klin Padiatr 2011;223(05):299-307

4 Sommers R, Stonestreet BS, Oh W, et al. Hemodynamic effects of delayed cord clamping in premature infants. Pediatrics 2012;129 (03):e667-e672

5 Garofalo M, Abenhaim HA. Early versus delayed cord clamping in term and preterm births: a review. J Obstet Gynaecol Can 2012;34 (06):525-531
6 Vural I, Ozdemir H, Teker G, Yoldemir T, Bilgen H, Ozek E. Delayed cord clamping in term large-for-gestational age infants: a prospective randomised study. J Paediatr Child Health 2019;55(05): 555-560

7 Andersson O, Lindquist B, Lindgren M, Stjernqvist K, Domellöf M, Hellström-Westas L. Effect of Delayed Cord Clamping on Neurodevelopment at 4 Years of Age: a Randomized Clinical Trial. JAMA Pediatr 2015;169(07):631-638

8 Wier LM, Witt E, Burgess J, Elixhauser A. Hospitalizations Related to Diabetes in Pregnancy, 2008: Statistical Brief \#102. In: Healthcare Cost and Utilization Project (HCUP) Statistical Briefs. Rockville (MD): Agency for Healthcare Research and Quality (US); December 2010

9 DeSisto CL, Kim SY, Sharma AJ. Prevalence estimates of gestational diabetes mellitus in the United States, Pregnancy Risk Assessment Monitoring System (PRAMS), 2007-2010. Prev Chronic Dis 2014; 11:E104

10 Nold JL, Georgieff MK. Infants of diabetic mothers. Pediatr Clin North Am 2004;51(03):619-637, viii

11 Mormile R, Vittori G, Vitale R, Squarcia U. Delayed cord clamping in full-term neonates: is it time for outlining exclusion criteria? J Pediatr Endocrinol Metab 2013;26(1-2):187-188

12 Emhamed MO, van Rheenen P, Brabin BJ. The early effects of delayed cord clamping in term infants born to Libyan mothers. Trop Doct 2004;34(04):218-222

13 Korkut S, Oğuz Y, Bozkaya D, et al. Evaluation of the effects of delayed cord clamping in infants of diabetic mothers. Am J Perinatol 2019. Doi: 10.1055/s-0039-169579

14 Vain NE, Satragno DS, Gorenstein AN, et al. Effect of gravity on volume of placental transfusion: a multicentre, randomised, noninferiority trial. Lancet 2014;384(9939):235-240

15 Adamkin DHCommittee on Fetus and Newborn. Postnatal glucose homeostasis in late-preterm and term infants. Pediatrics 2011; 127(03):575-579

16 Luchtman-Jones L, Schwartz AL, Wilson DB. The blood and hematopoietic system. Neonatal-perinatal medicine-diseases of the fetus and infant. 2002;2:1303-1360

17 McGrath RT, Glastras SJ, Hocking SL, Fulcher GR. Large-for-gestational-age neonates in type 1 diabetes and pregnancy: contribution of factors beyond hyperglycemia. Diabetes Care 2018;41(08): 1821-1828

18 American Academy of Pediatrics Subcommittee on Hyperbilirubinemia. Management of hyperbilirubinemia in the newborn infant 35 or more weeks of gestation. Pediatrics 2004;114(01): 297-316

19 Sankar MJ, Agarwal R, Deorari A, Paul VK. Management of polycythemia in neonates. Indian J Pediatr 2010;77(10): 1117-1121

20 Yaseen HA, Al-Najashi SS, Adel AA, Bahnassy AA, Al-Umran KU, AlFaraidy AA. Predictive factors and incidence of complications in apparently healthy full term infants of diabetic mothers. J Family Community Med 1999;6(02):37-42

21 Rincón D, Foguet A, Rojas M, et al. Tiempo de pinzamiento del cordón umbilical y complicaciones neonatales, un estudio prospectivo. An Pediatr (Barc) 2014;81(03):142-148

22 Chaparro CM, Neufeld LM, Tena Alavez G, Eguia-Líz Cedillo R, Dewey KG. Effect of timing of umbilical cord clamping on iron status in Mexican infants: a randomised controlled trial. Lancet 2006;367(9527):1997-2004

23 Ceriani Cernadas JM, Carroli G, Pellegrini L, et al. The effect of timing of cord clamping on neonatal venous hematocrit values and clinical outcome at term: a randomized, controlled trial. Pediatrics 2006;117(04):e779-e786

24 Spears RL, Anderson GV, Brotman S, et al. The effect of early versus late cord clamping on signs of respiratory distress. Am J Obstet Gynecol 1966;95(04):564-568 